\title{
Veto Values in Group Decision Making within MAUT Aggregating Complete Rankings Derived from Dominance Intensity Measures
}

\author{
Antonio Jiménez-Martín, Pilar Sabio and Alfonso Mateos \\ Departamento de Inteligencia Artificial, ETSI Informáticos, Universidad Politécnica de Madrid, \\ Campus de Montegancedo S/N, Boadilla del Monte, Spain
}

\begin{abstract}
We consider a group decision-making problem within multi-attribute utility theory, in which the relative importance of decision makers (DMs) is known and their preferences are represented by means of an additive function. We allow DMs to provide veto values for the attribute under consideration and build veto and adjust functions that are incorporated into the additive model. Veto functions check whether alternative performances are within the respective veto intervals, making the overall utility of the alternative equal to 0 , whereas adjust functions reduce the utilty of the alternative performance to match the preferences of other DMs. Dominance measuring methods are used to account for imprecise information in the decision-making scenario and to derive a ranking of alternatives for each DM. Specifically, ordinal information about the relative importance of criteria is provided by each DM. Finally, an extension of Kemeny's method is used to aggregate the alternative rankings from the DMs accounting for their relative importance.
\end{abstract}

\section{INTRODUCTION}

The additive model is considered a valid approach in many practical situations for the reasons described in (Raiffa, 1982) and (Stewart, 1996). Its functional form is

$$
u\left(A_{i}\right)=\sum_{j=1}^{n} w_{j} u_{j}\left(x_{i j}\right)
$$

where $x_{i j}$ is the performance over the attribute (or criterion) $X_{j}$ for the alternative $A_{i}, u_{j}$ is the component utility function and $w_{j}$ is the weight, respectively, for attribute $X_{j}$. Note that $\sum_{j=1}^{n} w_{j}=1$ and $w_{j} \geq 0$.

The additive model is a compensatory model in the sense that poor performance for an attribute can be compensated by good performances for other attributes.

For some multicriteria decision analysis (MCDA) problems and certain attributes, however, DMs may find it convenient to provide a veto value that identifies attribute performances that rule out the alternative regardless of the value taken in the other attributes. In a group decision-making context, moreover, more than one DM could provide different veto values for different attributes.

For example, let us consider a couple who decide to buy a home, so both have veto power. They identify several criteria for selecting the house, like the price, the location, the size or the age. One of the two might rule out any house smaller than $40 \mathrm{~m}^{2}$, regardless of house price, location and age, whereas the other might rule out any smaller than $60 \mathrm{~m}^{2}$. Therefore, in this group decision-making scenario, a possible veto range would be $[0,40]$, ruling out the purchase of any house smaller than $40 \mathrm{~m}^{2}$, and a possible adjust range would be $(40,60)$, decreasing the utility of the respective house to account for the DM veto values.

The veto concept has been variously considered as a real-world approach for representing the limits of DM preferences in the literature.To establish these preferences the veto threshold is represented as a quantifiable measure, which becomes an important tool in multicriteria and group decision-making.

In social theory, the concept of veto is justified by the prudence axiom enunciated by Arrow and Raynaud (Arrow and Raynaud, 1986), whose main idea is that it is not prudent to accept highly conflicting alternatives that may result in vulnerable decisions. Regarding the previous axiom, Moulin defines the principle of proportional veto in a group of DMs (Moulin, 1981), according to which any subset has the right to veto a number of alternatives in proportion to the size of the subgroup.

In MCDM problems the concept of veto has been 
used for the management of non-compensatory meth$o d s$. In outranking methods the use of veto usually represents the intensity of preference of the minority (Roy and Slowinski, 2008). Nowak used ELECTREIII to build a multi-attribute ranking using preference thresholds to distinguish situations of strict and weak preference in stochastic dominance approaches (Novak, 2004). Later, Munda (2009) implemented a vetobased threshold using fuzzy set theory to represent qualitative information.

Moreover, additive compensatory methods have also incorporated the concept of veto. An example is the technique for order preference by similarity to ideal solution (TOPSIS) method (Yoon, 1980). The basic idea behind TOPSIS is that the selected alternative should be as close to the ideal solution as far from the anti-ideal solution. Both alternatives behave like veto thresholds, not in the strict sense of rejection of alternatives but as reference points for solving the decision-making problem.

(Bana e Costa et al., 2002) defines a multi-criteria approach for prohibiting alternatives based on the measuring attractiveness by a categorical based evaluation technique (MACBETH). The methodological basis of this technique is indirectly related to the concept of veto power used in the non-compensatory models.

In connection with research based on the power of veto, (Marichal, 2004) proposes to axiomatize individual indices to valuate when each criterion behaves as a veto or for an aggregation by means of the Choquet integral. These indices make it possible to identify and measure the impact or trend of each criterion within the overall evaluation of the alternatives. In (Liginlala and Ow, 2006) the same idea of the effects of veto is used, expressing degrees of conjunction, disjunction, veto and approval given by the indices through fuzzy analysis measures, which represent a risk tolerance measure of the DM.

More recently, Daher and Almeida (Daher and Almeida, 2012) developed an additive group preference model that incorporates a utility reduction factor. DMs express their preferences in terms of a ranking of alternatives and are able to make an informed veto by providing information about the undesirable or unacceptable ranking of some alternatives. The ranking veto is achieved by using a reduction factor on the global utility of the alternatives.

In this paper we consider that veto values provided by DMs are applicable on alternative performances rather than on alternative rankings. We propose an additive multi-attribute value model accounting for the concepts of veto and adjust ranges. Different DMs with veto power identify a veto value on each attribute to express the limits of their preferences. They constitute the basis for constructing veto and adjust ranges, thus extending the classical additive model.

All DMs provide veto values, but the corresponding veto will be effective for only the most important DMs. These veto values are used to build a veto interval, whereas veto values corresponding to the least important DMs are used to build an adjust function that reduces component utilities to match the preferences of other DMs.

The extension of the additive multi-attribute value model to account for veto and adjust ranges is provided in Section 2. First, a veto and an adjust function is defined on the basis of the veto values provided by DMs. Then dominance intensity methods are introduced and used to derive a ranking of alternatives for each DM in Section 2.1. Finally, we aggregate the ranking from the different DMs to derive a consensus ranking in Section 2.2. Some conclusions are provided in Section 3.

\section{ADDITIVE MULTI-ATTRIBUTE VALUE MODEL ACCOUNTING FOR VETO}

We consider a set of $k$ DMs, denoted by $D M_{l}, l=$ $1, \ldots, k$, whose relative importance is known and denoted by $w_{D M_{i}}$. Without loss of generality we assume that the most important DM is $D M_{1}$, followed by $D M_{2}$, and so on until $D M_{k}$. Consequently, $w_{D M_{1}} \geq$ $w_{D M_{2}} \geq \ldots \geq w_{D M_{k}}$, and $\sum_{l} w_{D M_{l}}=1$.

How to measure the weights of DMs in a group decision-making context is an interesting research topic. (Yue, 2011) provides a brief overview of approaches proposed by different authors to determine the weights of DMs. Morever, a new approach based on an extended TOPSIS method is also proposed.

All DMs are allowed to provide veto values, but the corresponding veto will be effective for only the $r$ most important DMs, $r \leq k$. Veto values corresponding to the $k-r$ remaining DMs will be partially taken into account, as described later.

We consider a decision-making problem with $m$ alternatives $\left\{A_{1}, \ldots, A_{m}\right\}$ and $n$ attributes $\left\{X_{1}, \ldots, X_{n}\right\}$. DM preferences are modeled by an additive multiattribute utility function, see Eq (1).

Ordinal information about weights is available and each $D M_{l}$ provides an attribute importance ranking, arranged in descending order from the most to the least important attribute:

$$
\mathbf{w}^{l} \in W=\left\{\mathbf{w}^{l}=\left(w_{1}^{l}, \ldots, w_{n}^{l}\right) \mid w_{1}^{l} \geq \ldots \geq w_{n}^{l} \geq 0\right\},
$$


and $\sum_{i=1}^{n} w_{i}^{l}=1$.

Many methods accounting for ordinal information on weights and alternative values/utilities within MAVT/MAUT can be found in the literature (Sarabando and Dias, 2010; Punkka and Salo, 2013; Aguayo et al., 2014; Punkka and Salo, 2014).

We denote by $v_{j}^{l}$ the veto threshold provided by the $l$-th DM for the attribute $X_{j}$, i.e. the $l$-th DM wants the alternative performances to be equal to or greater (lower) than $v_{j}^{l}$ if an increasing (decreasing) component utility function is associated with attribute $X_{j}$. Consequently, the veto interval for the $l$-th DM is, $\left(0, v_{j}^{l}\right]$ in attribute $X_{j}$. For simplicity's sake, we will consider from now on that component utility functions are increasing.

A veto range can then be identified in each attribute $\left[v_{j}^{L}, v_{j}^{U}\right]$, where $v_{j}^{L}=r_{j}^{L}$, being $\left[r_{j}^{L}, r_{j}^{U}\right]$ the attribute range, and $v_{j}^{U}=\max _{l=1, ., r}\left\{v_{j}^{l}\right\}$, i.e. the highest veto value for attribute $X_{j}$ for the $r$ most important DMs.

We build an adjust range for each attribute $X_{j}$, $\left(a_{j}^{L}, a_{j}^{U}\right]$, with $a_{j}^{L}=v_{j}^{U}=\max _{l=1, \ldots, r}\left\{v_{j}^{l}\right\}$, i.e. the highest veto value for attribute $X_{j}$ for the $r$ most important DMs, and $a_{j}^{U}=\max _{l=1, \ldots, k}\left\{v_{j}^{l}\right\}$, i.e. the highest veto value for attribute $X_{j}$ considering all DMs.

We add the above information to the additive multi-attribute utility function by means of the following functions:

- $v\left(A_{i}\right)$ is the veto function that checks if the performances for a given $A_{i}$ are within the respective veto intervals:

$$
\begin{gathered}
v\left(A_{i}\right)=\prod_{j=1}^{n} v_{j}\left(A_{i}\right), \\
\text { with } v_{j}\left(A_{i}\right)=\left\{\begin{array}{ll}
1, & \text { if } x_{i j}>v_{\dot{j}}^{U} \\
0, & \text { if } x_{i j} \leq v_{j}^{U}
\end{array} .\right.
\end{gathered}
$$

Note that $v\left(A_{i}\right)=0$ if at least one performance is within the veto interval for the corresponding attribute.

- $d_{j}\left(A_{i}\right)$ is the adjust function that decreases the utility associated with the alternative performances within the corresponding adjust range. One possible approach is to apply a linear adjust function:

$$
d_{j}\left(A_{i}\right)=\left\{\begin{array}{cc}
1 & \text { if } x_{i j}>a_{j}^{U} \\
\frac{x_{i j}-a_{j}^{L}}{a_{j}^{U}-a_{j}^{L}} & \text { if } a_{j}^{L}<x_{i j} \leq a_{j}^{U} \\
0 & \text { if } x_{i j} \leq a_{j}^{L}
\end{array} .\right.
$$

However, we believe that the veto values for the $k-r$ less important DMs should be added by means of this adjust function. Veto values provided by the $k-r$ DMs may be within the adjust interval. In this case, we use this information to build a piecewise linear function.

For example, let us assume that the adjust range is $[20,50], 50$ being the highest veto value provided by the DMs. Then, if three of the $k-r$ less important DMs have provided the veto values 23,28 and 35 , i.e. veto values within the adjust range, then the adjust function shown in Fig. 1 could be plotted accounting for the above information.

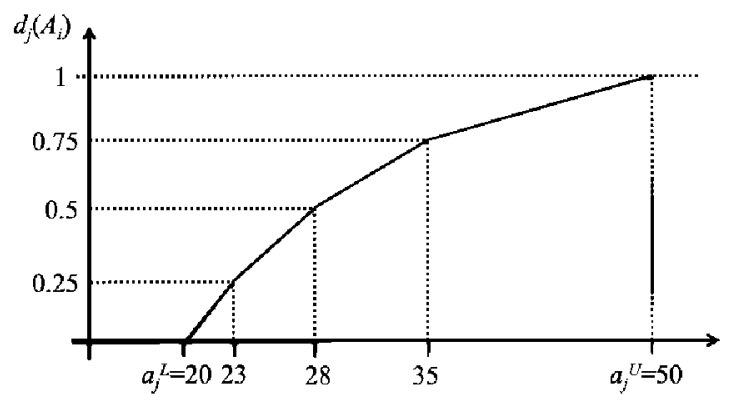

Figure 1: Example of adjust function.

The adaptation of the additive multi-attribute utility function to account for the veto and adjust functions would be as follows:

$$
u^{l}\left(A_{i}\right)=\left[\sum_{j=1}^{n} u_{j}\left(x_{i j}\right) w_{j}^{l} d_{j}\left(A_{i}\right)\right] \times v\left(A_{i}\right)
$$

This expression would then be used to derive a ranking of the alternative under consideration for each DM that should be aggregated taking into account the relative importance of DMs to reach a consensus ranking.

In the decision-making scenario under consideration, however, we have assumed ordinal information about weights, i.e. each DM has provided an attribute importance ranking, $\mathbf{w}^{l}$. In the next section we describe how to derive an alternatives ranking on the basis of the available ordinal information using dominance measuring methods, which are based on the notion of pairwise dominance. Then we review methods for aggregating rankings and select the best one for our decision-making scenario, in which complete rankings are available as well as their relative importance.

\subsection{Deriving a Ranking of Alternatives for each DM}

A recent approach for dealing with imprecise information, such as ordinal information about weights, is 
to compute different measures of dominance to derive a ranking of alternatives (Ahn and Park, 2008; Mateos et al., 2012; Jiménez et al., 2013; Aguayo, 2014; Mateos et al., 2014). These approaches known as dominance measuring methods (DMMs). DMMs are based on the computation of a dominance matrix, $D$, including pairwise dominance values, which are leveraged in different ways to derive measures of dominance to rank the alternatives under consideration.

The dominance matrix for the $l$-th DM, $D^{l}$, can be defined as follows:

$$
D^{l}=\left(\begin{array}{ccccc}
- & D_{12}^{l} & \cdots & D_{1(m-1)}^{l} & D_{1 m}^{l} \\
D_{21}^{l} & - & \cdots & D_{2(m-1)}^{l} & D_{2 m}^{l} \\
D_{31}^{l} & D_{32}^{l} & \cdots & D_{3(m-1)}^{l} & D_{3 m}^{l} \\
\vdots & \vdots & \vdots & \vdots & \vdots \\
D_{m 1}^{l} & D_{m 2}^{l} & \cdots & D_{m(m-1)}^{l} & -
\end{array}\right)
$$

where

$$
\begin{array}{cc}
D_{k s}^{l}= & \min \left\{u^{l}\left(A_{k}\right)-u^{l}\left(A_{s}\right)\right\} \\
\text { s.t. } & w_{1}^{l} \geq \ldots \geq w_{n}^{l} \geq 0
\end{array}
$$

in our decision-making scenario accounting for veto information, see $\mathrm{Eq}$ (2), and for ordinal information about weights.

Note that given two alternatives $A_{k}$ and $A_{s}$, alternative $A_{k}$ dominates $A_{s}$ if $D_{k s}^{l} \geq 0$, and there exists at least one combination of weights such that the overall value of $A_{k}$ is strictly greater than that of $A_{s}$. This concept of dominance is called pairwise dominance.

The optimization problems to be solved to derive pairwise dominance values are linear optimization problems. Consequently, they can be? efficiently solved using the simplex method. Note that ordinal information could also be considered in the component utilities, i.e., providing a ranking of the alternatives and also of the difference between the values of consecutive alternatives for each attribute, as in (Sarabando and Dias, 2010) or (Aguayo, 2014; Aguayo et al., 2014). Then the resulting optimization problem would likewise be linear.

As already mentioned, the dominance matrix, $D^{l}$, including pairwise dominance values, is used in different ways to derive measures of dominance to rank the alternatives under consideration in the DMMs. For instance, in (Mateos et al., 2014) a DMM is proposed, which derives a global dominance intensity index to rank alternatives on the basis that

$$
\begin{gathered}
D_{k s}^{l} \leq \mathbf{w}^{l T}\left(\mathbf{u}\left(A_{k}\right)-\mathbf{u}\left(A_{s}\right)\right) \leq-D_{s k}^{l}, \\
\forall \mathbf{w}^{l} \mid w_{1}^{l} \geq \ldots \geq w_{n}^{l} \geq 0,
\end{gathered}
$$

with $\mathbf{u}\left(A_{i}\right)=\left(u_{1}\left(x_{i 1}\right) \times d_{1}\left(A_{i}\right), \ldots, u_{n}\left(x_{i n}\right) \times d_{n}\left(A_{i}\right)\right)$.

The DMM is implemented as follows:
1. If $D_{k s}^{l} \geq 0$, then alternative $A_{k}$ dominates $A_{s}$, and the dominance intensity of $A_{k}$ over $A_{s}\left(D I_{k s}^{l}\right)$ is 1 , i.e., $D I_{k s}^{l}=1$.

Else $\left(D_{k s}^{l}<0\right)$ :

- If $D_{s k}^{l} \geq 0$, then alternative $A_{l}$ dominates $A_{k}$, and $D I_{k s}^{l}=0$.

- Else $\left(D_{s k}^{l}<0\right), D I_{k s}^{l}=\frac{-D_{s k}^{l}}{-D_{s k}^{l}-D_{k s}^{l}}$.

2. Calculate a global dominance intensity $(G D I)$ for each alternative $A_{k}$, i.e. $G D I_{k}^{l}=\sum_{s=1, s \neq k}^{m} D I_{k s}^{l}$, and rank the alternatives according to them.

The performance of this method was compared in (Mateos et al., 2014) with other existing approaches (surrogate weighting methods, which select a weight vector from a set of admissible weights to represent the set (Stillwell et al., 1981); modified classical decision rules (Salo and Hamalainen, 2001; Puerto et al., 2000), and the DMM proposed in (Ahn and Park, 2008)), where ordinal information represents imprecision concerning weights.

Monte Carlo simulation was used to demonstrate that there is, according to the paired-samples $t$-test, no significant difference between the DMM used in this paper and the rank-order centroid weights method (ROC), a surrogate weighting method, and that they outperform the other methods in terms of average hit ratios and rank-order correlations. However, ROC can be only applied when ordinal relations regarding attribute weights are provided, whereas DMM is more generally applicable since it can also be used when the imprecision concerning weights or even value functions is represented in other ways, for example by interval values, probability distributions or even fuzzy numbers.

Note that the described dominance measuring method is used to derive $k$ rankings of alternatives, i.e a ranking for each DM.

\subsection{Aggregating the Ranking of Alternatives}

Different methods for aggregating rankings can be found in the literature by different authors. (Lin, 2010) discusses three classes of methods, namely distribution-based, heuristic, and stochastic optimization search.

The original Thurstone scaling and its extensions (Thurstone, 1931; Green, 1978) represent the first class of methods that are most appropriate for aggregating many short ranked lists. Heuristic algorithms and stochastic search methods are applicable for aggregating a small number of long lists. 
Heuristic algorithms are deterministic, ranging from simple arithmetic averages of ranks (Borda's methods, (Borda, 1981)) to Markov chains and stationary distributions, in which only pairwise ranking information is used (Dwork et al., 2001; DeConde et al., 2006).

Stochastic search algorithms, on the other hand, aim at maximizing a particular criterion. They are usually distance measure dependent, and Kemeny optimal aggregation (which optimizes the average Kendall distances between a candidate aggregate list and each of the input lists) is an example. However, it is widely recognized that computing the Kemeny optimal aggregate is NP-hard even when the number of ranked lists to be aggregated is small. Stochastic search algorithms based on the cross entropy Monte Carlo approach, such as the order explicit algorithm (Lin and Ding, 2009), provide an alternative for finding an optimal solution while circumventing the combinatorial nature of the problem. Instead of imposing a discrete uniform distribution on all the potential solutions, an iterative importance sampling technique is utilized to slowly tighten the net to place most distributional mass on the optimal solution and its neighbors. Extensive simulation studies were performed to assess the performance of the method, leading to satisfactory results.

Besides, (Niu et al., 2013) divides rank aggregation methods into two categories according to the way in which rank information is used: explicit and implicit rank aggregation methods. Explicit methods directly utilize rank information to define the ranking function or the objective function, whereas, for $\mathrm{im}$ plicit methods, other information, such as pairwise preference or list-wise ranking is first gathered based on the rank information and then leveraged for rank aggregation.

Note also that the above methods can be categorized taking into account whether they assume a complete, partial or top $k$-rank ranking of the alternatives and whether the analysis includes the relative importance of the rankings.

In our decision-making scenario, complete rankings and the relative importance of such rankings (relative importance of DMs) are available. Moreover, the values that lead to the corresponding rankings (global dominance intensities) are also available. The only aggregation methods that exploit all the above information is the Kemeny method (Kemeny, 1959) and its extensions.

As already mentioned, Kemeny optimal aggregation optimizes the average Kendall distances between a candidate aggregate ranking and each of the input rankings. As computing the Kemeny optimal aggre- gate is NP-hard even when the number of ranked lists to be aggregated is small, we have used the order explicit algorithm (OEA)(Lin and Ding, 2009) to solve the combinatorial problem under consideration.

OEA uses a global optimization technique, called the cross-entropy Monte Carlo method, which searches iteratively for an optimal list that minimizes a criterion, the sum of weighted distances between the candidate (aggregate) list and each of the input ranked lists. The method is, however, general and amenable to any other optimization criterion. A modified Kendall's tau measure and the Spearman's footrule, as described in (Fagin et al., 2003), are used to measure the distance between two ranked lists.

\section{AN ILLUSTRATIVE EXAMPLE}

We consider five DMs whose relative importance is $w_{D M_{1}}=0.35 \geq w_{D M_{2}}=0.25 \geq w_{D M_{3}}=0.2 \geq w_{D M_{4}}=$ $0.1=w_{D M_{5}}=0.1$. Seven alternatives $\left\{A_{1}, \ldots, A_{7}\right\}$ will be analyzed on the basis of four attributes $\left\{X_{1}, \ldots, X_{4}\right\}$, whose ranges are $[0,100]$ in all cases.

The corresponding veto will be effective for only the three most important DMs. Table 1 shows the veto values provided by the DMs. Note that except for $D M_{1}$ DMs do not provide veto values for all attributes and the only veto for attribute $X_{4}$ is provided by $D M_{1}$.

Table 1: Veto values for DMs

\begin{tabular}{|c|c|c|c|c|}
\hline & $X_{1}$ & $X_{2}$ & $X_{3}$ & $X_{4}$ \\
\hline$D M_{1}$ & 20 & 15 & 10 & 20 \\
\hline$D M_{2}$ & 15 & 10 & 5 & - \\
\hline$D M_{3}$ & 25 & 10 & - & - \\
\hline$D M_{4}$ & 30 & 25 & - & - \\
\hline$D M_{5}$ & 27 & 10 & - & - \\
\hline
\end{tabular}

Each DM expresses the relative importance of the attributes under consideration, see Table 2 . We assume that the four component utility functions are linear and increasing in the attribute ranges, $[0,100]$, for the five DMs.

Table 2: Relative importance of attributes for DMs.

\begin{tabular}{|l|c|}
\hline & Relative importance of attributes \\
\hline$D M_{1}$ & $w_{1} \geq w_{2} \geq w_{3} \geq w_{4}$ \\
\hline$D M_{2}$ & $w_{1} \geq w_{3} \geq w_{2} \geq w_{4}$ \\
\hline$D M_{3}$ & $w_{3} \geq w_{1} \geq w_{2} \geq w_{4}$ \\
\hline$D M_{4}$ & $w_{1} \geq w_{3} \geq w_{4} \geq w_{2}$ \\
\hline$D M_{5}$ & $w_{2} \geq w_{1} \geq w_{4} \geq w_{3}$ \\
\hline
\end{tabular}

Table 3 shows the alternative performances for the four attributes under consideration as well as the veto and adjust ranges for each attribute. 
Table 3: Alternative performances and veto and adjust ranges.

\begin{tabular}{|c|c|c|c|c|}
\hline & $X_{1}$ & $X_{2}$ & $X_{3}$ & $X_{4}$ \\
\hline$A_{1}$ & 35 & 60 & 40 & 35 \\
\hline$A_{2}$ & 20 & 20 & 40 & 65 \\
\hline$A_{3}$ & 26 & 27 & 30 & 94 \\
\hline$A_{4}$ & 40 & 35 & 50 & 63 \\
\hline$A_{5}$ & 35 & 85 & 10 & 33 \\
\hline$A_{6}$ & 60 & 17 & 20 & 45 \\
\hline$A_{7}$ & 27 & 65 & 20 & 45 \\
\hline \hline Veto range & {$[0,25]$} & {$[0,15]$} & {$[0,10]$} & {$[0,20]$} \\
\hline Adjust range & $(25,30]$ & $(15,25]$ & - & - \\
\hline
\end{tabular}

The adjust functions for attributes $X_{1}$ and $X_{2}$ are shown in Fig. 2. Note that the adjust function for attribute $X_{2}$ is a linear function since none of the DMs provided a veto value within the adjust range, whereas the adjust function for attribute $X_{1}$ is a piecewise utility function since the veto value 27 corresponding to $D M_{5}$ is within the adjust range and assigned a value 0.5 .
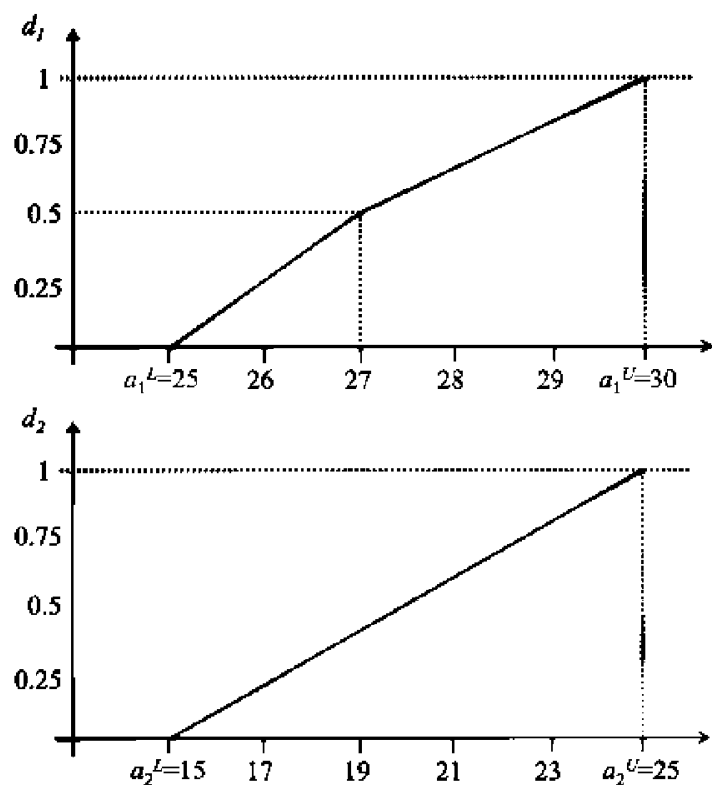

Figure 2: Adjust functions for attributes $X_{1}$ and $X_{2}$.

Table 4 shows the values output by the veto and adjust function for the alternative performances included in Table 3. The performances of alternatives $A_{2}$ and $A_{5}$ for attributes $X_{1}$ and $X_{3}$, respectively, are vetoed. The utility for those alternatives will be 0 , see Eq. 2, and both will always be the two worst-ranked alternatives and will, therefore, be worst ranked in the consensus ranking. Consequently, we have omitted these alternatives from further analyses.

Besides, adjust functions decrease the component utility associated with alternatives $A_{3}$ and $A_{7}$ for at- tribute $X_{1}$ and with alternatives $A_{2}$ and $A_{6}$ for attribute $X_{2}$.

Table 4: Veto and adjust values.

\begin{tabular}{|c|c|c|c|}
\hline & $v\left(A_{i}\right)$ & $d_{1}\left(A_{i}\right)$ & $d_{2}\left(A_{i}\right)$ \\
\hline$A_{1}$ & 1 & 1 & 1 \\
\hline$A_{2}$ & 0 & 0 & 0.5 \\
\hline$A_{3}$ & 1 & 0.25 & $\mathbf{1}$ \\
\hline$A_{4}$ & 1 & 1 & 1 \\
\hline$A_{5}$ & 0 & 1 & 1 \\
\hline$A_{6}$ & 1 & 1 & 0.2 \\
\hline$A_{7}$ & 1 & 0.5 & $\mathbf{1}$ \\
\hline
\end{tabular}

Optimization problems can be solved using the simplex method to derive the dominance matrices corresponding to the five DMs. Dominance matrices are as follows:

$$
\begin{aligned}
& D^{1}=\left(\begin{array}{ccccc}
- & .031 & .05 & -.248 & .066 \\
-.306 & - & -.334 & -.532 & -.224 \\
-.099 & .076 & - & -.198 & -.017 \\
-.171 & -.07 & -.149 & - & -.075 \\
-.214 & -.035 & -.281 & -.463 & -
\end{array}\right) \\
& D^{2}=\left(\begin{array}{ccccc}
- & .031 & .075 & -.248 & .066 \\
-.284 & - & -.334 & -.532 & -.116 \\
-.033 & .076 & - & -.198 & .088 \\
-.171 & -.022 & -.149 & - & -.05 \\
-.214 & -.035 & -.264 & -.463 & -
\end{array}\right) \text {, } \\
& D^{3}=\left(\begin{array}{ccccc}
- & .031 & -.099 & -.024 & .116 \\
-.237 & - & -.266 & -.216 & -.116 \\
-.033 & .076 & - & .05 & .088 \\
-.199 & -.099 & -.299 & - & -.05 \\
-.207 & -.099 & -.299 & -.231 & -
\end{array}\right) \text {, } \\
& D^{4}=\left(\begin{array}{ccccc}
- & -.067 & -.143 & -.248 & .066 \\
-.284 & - & -.334 & -.532 & -.069 \\
.045 & .075 & - & -.198 & .111 \\
-.104 & -.072 & -.149 & - & -.037 \\
-.214 & -.173 & -.281 & -.463 & -
\end{array}\right) \text {, } \\
& D^{5}=\left(\begin{array}{ccccc}
- & .008 & -.045 & .072 & -.049 \\
-.329 & - & -.207 & -.148 & -.378 \\
-.249 & .035 & - & .058 & -.298 \\
-.564 & -.235 & -.315 & - & -.613 \\
-.082 & -.035 & -.111 & .037 & -
\end{array}\right) .
\end{aligned}
$$

Note that the alternatives corresponding to the rows and columns of the above dominance matrices are $A_{1}$, $A_{3}, A_{4}, A_{6}$ and $A_{7}$, since $A_{2}$ and $A_{5}$ were removed from the analysis.

The dominance measuring method described in the previous section is then applied to compute global dominance intensities (GDIs) for each DM on the basis of which to derive the corresponding ranking of the considered alternatives.

Table 5 shows the GDIs associated with each alternative, whereas Table 6 shows the resulting rankings of alternatives for the DMs under consideration. 
Table 5: Global dominance intensities.

\begin{tabular}{|l|c|c|c|c|c|}
\hline & $D M_{1}$ & $D M_{2}$ & $D M_{3}$ & $D M_{4}$ & $D M_{5}$ \\
\hline$A_{1}$ & 5.4 & 5.4 & 5.1 & 4.1 & 5.5 \\
\hline$A_{3}$ & 2.2 & 2.3 & 2.8 & 3.0 & 2.7 \\
\hline$A_{4}$ & 4.3 & 4.4 & 5.7 & 5.4 & 4.4 \\
\hline$A_{6}$ & 4.9 & 5.0 & 3.6 & 5.1 & 2.4 \\
\hline$A_{7}$ & 3.1 & 2.9 & 2.7 & 2.4 & 5.0 \\
\hline
\end{tabular}

Table 6: Alternative rankings for DMs.

\begin{tabular}{|c|c|c|c|c|c|}
\hline & $D M_{1}$ & $D M_{2}$ & $D M_{3}$ & $D M_{4}$ & $D M_{5}$ \\
\hline $1^{\text {st }}$ & $A_{1}$ & $A_{1}$ & $A_{4}$ & $A_{4}$ & $A_{1}$ \\
\hline $2^{\text {nd }}$ & $A_{6}$ & $A_{6}$ & $A_{1}$ & $A_{6}$ & $A_{7}$ \\
\hline $3^{\text {rd }}$ & $A_{4}$ & $A_{4}$ & $A_{6}$ & $A_{1}$ & $A_{4}$ \\
\hline $4^{\text {th }}$ & $A_{7}$ & $A_{7}$ & $A_{3}$ & $A_{3}$ & $A_{3}$ \\
\hline $5^{\text {th }}$ & $A_{3}$ & $A_{3}$ & $A_{7}$ & $A_{7}$ & $A_{6}$ \\
\hline
\end{tabular}

Finally, Lin and Din's method (OEA) is used to aggregate the rankings in Table 6 also taking into account the relative importance of such rankings (relative importance of DM), $w_{D M_{1}}=0.35 \geq w_{D M_{2}}=$ $0.25 \geq w_{D M_{3}}=0.2 \geq w_{D M_{4}}=0.1=w_{D M_{5}}=0.1$, to derive the consensus ranking shown in Table 7.

Table 7: Consensus ranking.

\begin{tabular}{|c|c|}
\hline & Alternative \\
\hline $\mathbf{1}^{\text {st }}$ & $A_{1}$ \\
\hline $2^{\text {nd }}$ & $A_{6}$ \\
\hline $3^{\text {rd }}$ & $A_{4}$ \\
\hline $4^{\text {th }}$ & $A_{7}$ \\
\hline $5^{\text {th }}$ & $A_{3}$ \\
\hline
\end{tabular}

Note that alternative $A_{1}$ is best ranked in the consensus ranking, followed by $A_{6}$ and $A_{4}$. Alternative $A_{1}$ was best ranked by the two most important DMs $\left(D M_{1}\right.$ and $D M_{2}$ ) and by $D M_{5}$, whereas it was placed second by $D_{3}$. Although alternative $A_{4}$ was first ranked by $D M_{3}$ and $D M_{4}$, the relative importance of both DMs in the consensus is only 0.3 , and $A_{4}$ is placed third by the other three DMs.

Note also that the ranking for DM1 matches the consensus ranking. A sensitivity analysis was carried out to identify the weight of $D M_{1}$ for which the consensus ranking differed. To do this, $w_{D M 1}$ is progressively decreased, the remaining weights proportionally updated and the consensus ranking recomputed. The value for $w_{D M 1}$ that makes the consensus ranking different than the $D M_{1}$ 's ranking is 0.2166 , and the corresponding consensus ranking is $A_{1}, A_{4}, A_{6}$, $A_{7}$ and $A_{3}$. We found that $A_{1}$ is the best-ranked alternative no matter what the value of $w_{D M 1}$ is.

\section{CONCLUSIONS}

In this paper we have extended the additive multiattribute utility model to incorporate the concept of veto as an approximation to real situations to represent the limits of the DM preferences.

Although all DMs are allowed to provide veto values, the corresponding vetoes are effective for only the most important DMs. They are used to define veto ranges. Veto values corresponding to the other less important DMs are partially taken into account, leading to the construction of adjust ranges. A veto and an adjust function are then incorporated into the additive model.

A dominance measuring method is used to account for imprecise information (ordinal information about weights) in the decision-making scenario and to derive the ranking of alternatives, whose performance has been analyzed in the literature using Monte Carlo simulation techniques.

Finally, Kemeny's method was selected to aggregate the alternative rankings from the DMs since it is the method that best fits the decision-making information, i.e. both complete rankings (and the respective global dominance intensities) and their relative importance are available.

As computing the Kemeny optimal aggregate is NP-hard, a stochastic search method, the order explicit algorithm proposed by Lin and Din, was used to solve this combinatorial problem.

We propose the possibility of using trapezoidal fuzzy numbers to aggregate group preferences regarding the attribute weights as a future research line. Then, a fuzzy dominance matrix would be computed and a dominance-measuring method accounting for it would be used to derive a fuzzy ranking of alternatives for each DM. Finally, a fuzzy ranking aggregation process would be performed to derive a consensus ranking.

Another research line is the study of parameter $r$, i.e. the number of DMs whose vetoes are effective on the basis of a threshold on their relative importance.

\section{ACKNOWLEDGEMENTS}

The paper was supported by the Spanish Ministry of Science and Innovation project MTM2011-28983C03-03. 


\section{REFERENCES}

Aguayo, E. (2014). Dominance Intensity Methods for Ranking of Alternatives in MCDM with Imprecise Information. $\mathrm{PhD}$ thesis, Universidad Politécnica de Madrid.

Aguayo, E., Mateos, A., and Jiménez-Martín, A. (2014). A new dominance intensity method to deal with ordinal information about a DM's preferences within MAVT. Knowledge-Based Systems, 69:159-169.

Ahn, B. and Park, K. (2008). Comparing methods for multiattribute decision making with ordinal weights. Computers and Operations Research, 35:1660-1670.

Arrow, K. J. and Raynaud, H. (1986). Social Choice and Multicriterion Decision Making. MTT Press, Cambridge.

Bana e Costa, C., Corrêa, E., DeCorte, J. M., and Vansnick, J. C. (2002). Faciliting bid evalutation in public call for tenders: a social-technical approach. OMEGA. The International Journal of Management Science, 30:227-242.

Borda, J. (1981). Memoire sur les elections au scrutin. Histoire de l'Academie des Sciences.

Daher, S. and Almeida, A. (2012). The use of ranking veto concept to mitigate the compensatory effects of additive aggregation in group decisions on a water utility automation investment. Group Decision and Negotiation, 21:185-204.

DeConde, R., Hawley, S., Falcon, S., Clegg, N., Knudsen, B., and Etzioni, R. (2006). Combining results of microarray experiments: a rank aggregation approach. Statistical Applications in Genetics and Molecular Biology, 5:5-15.

Dwork, C., Kumar, R., Naor, M., and Sivakumar, D. (2001). Rank aggregation methods for the web. In Proceedings of the 10th International World Wide Web Conference.

Fagin, R., Kumar, R., and Sivakumar, D. (2003). Comparing top $\mathrm{k}$ lists. SIAM Journal of Discrete Mathematics, $17: 134-160$

Green, P. (1978). Research for Marketing Decisions. Prentice-Hall, New Jersey.

Jiménez, A., Mateos, A., and Sabio, P. (2013). Dominance intensity measure within fuzzy weight oriented MAUT: An application. OMEGA. The International Journal of Management Science, 41(2):397-405.

Kemeny, J. (1959). Mathematics without numbers. Daedalus, 88:577-591.

Liginlala, D. and Ow, T. T. (2006). Modeling attitude to risk in human decision processes: An application of fuzzy measures. Fuzzy Sets and Systems, 157:3040-3054.

Lin, S. (2010). Rank aggregation methods. Wiley Interdisciplinary Reviews: Computational Statistics, 2:555570 .

Lin, S. and Ding, J. (2009). Integration of ranked lists via cross entropy Monte Carlo with applications to mRNA and microRNA studies. Biometrics, 65:9-18.

Marichal, J. L. (2004). Tolerant or intolerant character of interacting criteria in aggregation by the Choquet integral. European Journal of Operations Research, 155:771-791.
Mateos, A., Jiménez, A., and Blanco, J. (2012). Measuring method performance under incomplete information about weights. Journal of Multicriteria Decision Analysis, 19(3-4):129-142.

Mateos, A., Jiménez-Martín, A., Aguayo, E., and Sabio, P. (2014). Dominance intensity measuring methods in MCDM with ordinal relations regarding weights. Knowledge-Based Systems, 70:26-32.

Moulin, H. (1981). The proportional veto principle. Review of Economic Studies, 48:407-416.

Niu, S., Lan, Y., Guo, J., and Cheng, X. (2013). Stochastic rank aggregation. In Proceedings of the Twenty-Ninth Conference on Uncertainty in Artificial Intelligence.

Novak, M. (2004). Preference and veto threshold in multicriteria analysis based on stochastic dominance. European Journal of Operations Research, 158:339-350.

Puerto, J., Mármol, A., Monroy, L., and Fernández, F. (2000). Decision criteria with partial information. IEEE Transaction in Operational Research, 7:51-65.

Punkka, A. and Salo, A. (2013). Preference programming with incomplete ordinal information. European Journal of Operational Research, 231(1):141-150.

Punkka, A. and Salo, A. (2014). Scale dependence and ranking intervals in additive value models under incomplete preference information. Decision Analysis, 11:83-104.

Raiffa, H. (1982). The Art and Science of Negotiation. Haarvard University Press, Cambridge.

Roy, B. and Slowinski, R. (2008). Handling effects of reinforced preference and counter-veto in credibility of outranking. European Journal of Operations Research, 188:185-190.

Salo, A. and Hamalainen, R. P. (2001). Preference ratio in multiattribute evaluation (PRIME)-elicitation and decision procedures under incomplete information. IEEE Transactions on Systems, Management and $C y$ bernetics: Part A, 31:533-545.

Sarabando, P. and Dias, L. (2010). Simple procedures of choice in multicriteria problems without precise information about the alternatives values. Computers and Operations Research, 37:2239-2247.

Stewart, T. (1996). Robustness of additive value function method in MCDM. Journal of Multicriteria Decision Analysis, 5:301-309.

Stillwell, W., Seaver, D., and Edwards, W. (1981). Comparison of weight approximation techniques in multiattribute utility decision making. Organizatorial Behaviour and Human Decision Processes, 28:62-77.

Thurstone, L. (1931). Rank order as a psychophysical method. Journal of Experimental Psychology, 14:187-201.

Yoon, K. (1980). System Selection by Multiple Attribute Decision Making. $\mathrm{PhD}$ thesis, Kansas State University.

Yue, Z. (2011). A method for group decision-making based on determining weights of decision makers using TOPSIS. Applied Mathematical Modelling, 35:19261936. 